\title{
DEPRESSÃO INFANTIL: PRODUÇÃO CIENTIFICA EM PERIÓDICOS NACIONAIS
}

Jéssica Gorrão, Caio Henrique Nobre Cabral, Carolina Morais Faria, Carolina Braga Borges, Gabriel Gonzaga dos Santos, Camélia Santina Murgo.

Universidade do Oeste Paulista - UNOESTE, Faculdade de Medicina, Presidente Prudente, SP. E-mail: camélia@unoeste.br.

\section{RESUMO}

O presente trabalho objetivou realizar um levantamento de artigos científicos indexados em periódicos nacionais na última década sobre a depressão infantil. Para a coleta dos materiais foram utilizadas as bases de dados eletrônicas Periódicos da Coordenação de Aperfeiçoamento de Pessoal de Nível Superior (CAPES); da Biblioteca Virtual em Saúde Brasil (BVS-BRASIL) e da Scientific Electronic Library Online (SciELO). No total, 48 artigos foram recuperados. A categorização dos dados possibilitou identificar que entre as temáticas destacadas estão a validação de instrumentos para medição da depressão, incidência de depressão, variáveis ambientais associadas, representações da criança sobre a depressão e relações entre depressão infantil e rendimento escolar. As comorbidades citadas foram obesidade infantil, ansiedade, distúrbios do sono, estresse e transtorno bipolar. A maior parte dos artigos encontrados trazem investigações realizadas nos contextos escolar, da saúde e institucional. Foi possível verificar que o tema vem sendo desenvolvido expressivamente nas ciências médica e psicológica.

Palavras-chave: Depressão Infantil, Comorbidades, Criança.

\section{ABSTRACT}

The present study aimed to perform a survey of scientific articles in national journals indexed in the last decade about childhood depression. The electronic databases of journals Coordination of Improvement of Higher Education Personnel (CAPES) were used to collect materials ; of Brazil Virtual Health Library ( VHL - BRAZIL) and Scientific Electronic Library Online ( SciELO ) . In total, 48 articles were retrieved from which created three categories of analysis : most recurrent themes, comorbidities and research settings. The categorization identified that among the outstanding issues are mentioned instrument validation, incidence of depression in children, associated environmental variables, representations of children on depression and relationships between childhood depression and school performance. Comorbidities were cited childhood obesity, anxiety, sleep disorders, stress and bipolar disorder. Most of the articles found bring investigations in school settings, health and institutional. It was possible to verify that the topic has been developed significantly in Psychological Science.

Key words: Childhood depression, comorbidity, Child. 


\section{INTRODUÇÃO}

Dentre os transtornos mentais com maior incidência, segundo a Organização Mundial de Saúde em relatório publicado em 2012, a depressão ocupou o terceiro lugar em 2004 e possivelmente ocupará o primeiro lugar em 2030 onde a estimativa é de que cerca de 350 milhões de pessoas sofram com a patologia. Especificamente em relação a depressão infantil, estudos apontam que dentro da população mundial cerca de cinco por cento (5\%) das crianças e adolescentes apresentem, em algum grau, sintomas depressivos. Diante desta constatação a presença dos sintomas depressivos em crianças e adolescentes se tornou alvo de estudos que buscam evidenciar sua presença, bem como definir de forma clara seus sintomas e verificar se existem diferenças entre suas manifestações na infância e a na idade adulta (WHO, 2012). .

A este respeito o DSM-5 (2014) acrescenta que em crianças e adolescentes que apresentam os sintomas depressivos, ao invés de demostrarem um humor triste e abatido pode aparecer um humor irritável ou rabugento o que, de certa forma, caracteriza a existência do transtorno depressivo em crianças, dificultando seu reconhecimento e diagnóstico. A sintomatologia especifica da depressão em crianças o Manual Diagnóstico menciona queda abrupta no rendimento escolar explicada pela dificuldade de concentração que a criança vivencia nos episódios depressivos. Vale ainda ressaltar, que em muitos casos, a presença de sintomas de depressão em crianças pode estar relacionada à sensação prolongada de desamparo, incapacidade de se divertir, sentimentos de falta de valor, fadiga entre outros sintomas (PAPALIA, 2009).

Nessa direção, Bahls (2002) afirma que com maior frequência que os adultos depressivos, crianças podem apresentar comorbidades, tais como, transtorno de ansiedade, transtorno bipolar, distúrbios do sono, entre outros. Além disso, a sintomatologia pode apresentar diferenças, mesmo entre as crianças, de acordo com a idade e o período de desenvolvimento em que a mesma se encontra. Há da mesma forma, para alguns autores, diferenciações dentro da sintomatologia apresentada por crianças segundo sua faixa etária.

Como principal referência para o diagnóstico da depressão em crianças tem sido utilizado os critérios contidos no DSM-5 (2014). Pelo fato de, as características particulares presentes nos sintomas depressivos estarem relacionadas com questões internas Cruvinel, Boruchovitch e Santos (2008a) mencionam como tendência que a própria criança possa ser a principal informante no que diz respeito às alterações e sintomas causados pela patologia o que valoriza a utilização de Inventários e escalas de autoavaliação. Analogamente tem se feito uso de técnicas de observação e entrevistas estruturadas e semiestruturadas no intuito de obter informações que permitam o diagnóstico.

Diversos estudos vêm sendo realizados no intuito de aprofundar os conhecimentos sobre a depressão na infância, sua provável etiologia, seus principais sintomas, manifestações e implicações para o desenvolvimento da criança. Dentre eles o estudo de Cruvinel e Boruchovitch (2011) buscou avaliar a regulação emocional de alunos de 3a e 4a série do ensino fundamental, com amostra composta por 54 alunos divididos em dois grupos, um (G1) com 27 crianças que apresentavam sintomas depressivos e outro (G2) com 27 crianças que não apresentavam. Foram utilizados como instrumentos o Inventário de Depressão Infantil - CDI (Kovacs, 1992) e a Entrevista com Pranchas para Avaliação da Regulação Emocional de Alunos do Ensino Fundamental - EPRE (Cruvinel e Boruchovitch, 2008). O resultado encontrado demonstrou que as crianças de ambos os grupos utilizavam estratégias semelhantes no cotidiano para lidar com as diversas emoções, porém as que faziam parte do G1 indicaram sentir mais frequentemente tristeza e raiva, além de dificuldades na percepção de emoções como tristeza, medo e alegria.

Em outro caso, o estudo realizado por Santos (2006) visava identificar e conhecer as principais características e queixas, tanto comportamentais como emocionais, de crianças atendidas por um serviço publico de Psicologia Infantil, por meio de dados coletados dos prontuários dos pacientes. Participaram deste estudo 129 crianças e adolescentes entre 2 e 17 
anos de ambos os sexos e diferentes níveis de escolaridade. Os dados obtidos, segundo a autora, apontam comportamentos que são característicos da depressão que podem indicar uma tendência ao aumento da depressão entre crianças apresentando sintomas depressivos a partir dos seis (6) anos de idade, além disso, alguns dados sobre a queixa dos pais indicam uma incidência significativa de desinteresse pela escola $(19,4 \%)$ e dificuldade nos relacionamentos sociais/familiares $(13,2 \%)$ que podem ter relação com comportamentos depressivos na Infância. Porém salientam a necessidade de serem realizadas mais pesquisas a respeito. (SANTOS, 2006)

Já Lima (2012) buscou verificar a existência de relação entre a compreensão de leitura e a escrita com os sintomas da depressão infantil entre alunos matriculados no ensino fundamental tendo como participantes 293 crianças com idades entre 7 e 11 anos de duas escolas públicas do interior de São Paulo. Foram utilizados como instrumentos o CDI, dois textos estruturados segundo os padrões tradicionais da técnica de Cloze e a Escala de Avaliação da Escrita (EAVE). Os resultados obtidos apontaram que crianças obtiveram maior pontuação no CDI tiveram pior compreensão de leitura e escrita. Considerando a diversidade de pesquisas já realizadas sobre a depressão infantil, foi delimitado o objetivo desse estudo, a saber, sistematizar as informações por meio dos conteúdos apresentados na última década sobre a temática $\mathrm{m}$ periódicos nacionais na intenção de identificar possíveis lacunas que favoreçam o delineamento de aspectos a serem explorados em novas investigações.

\section{METODO}

O procedimento de coleta de dados realizou-se por meio da busca de artigos científicos a partir de bases de dados eletrônicas brasileiras, as quais agrupam importantes revistas científicas. Trata-se, portanto, da base de dados dos Periódicos da Coordenação de Aperfeiçoamento de Pessoal de Nível Superior (CAPES); da Biblioteca Virtual em Saúde Brasil (BVS-BRASIL) e da Scientific Electronic Library Online (SciELO). Recuperou-se seiscentos e trinta e três artigos nos periódicos CAPES, trezentos e quarenta e sete na BVS-BRASIL, oitenta e oito no SciELO e análise dos artigos encontrados seguiram os seguintes critérios de inclusão: tratarem sobre a temática da depressão na infância e terem sido publicado no período de 2004 à 2017. Nesse sentido, a revisão constou de três etapas, sendo a primeira a definição dos descritores, a segunda a busca dos artigos nas bases de dados por meio dos descritores selecionados, e a terceira a classificação e análise dos artigos recuperados, considerando categorias, as quais foram construídas por meio de informações buscadas nos resumos, ou sob consulta aos textos completos obtendo como resultado final 48 artigos analisados.

\section{RESULTADOS}

Iniciando a análise dos trabalhos selecionados observou-se que a maior produção sobre a depressão infantil se concentrou entre os anos de 2014 e 2017 onde foram encontrados 26 (33,3 \%) artigos. Em contrapartida o período de menor produção esteve nos anos de 2012 e 2013 apresentando quatro (4,1\%). Entre os anos de 2004 a 2011 somaram-se 30 artigos. Considerando os artigos segundo os periódicos nos quais foram publicados foi possível também identificar a área que mais publicou sobre a depressão infantil foi à das ciências médicas, seguida da área da Psicologia . As revistas com publicações foram Jornal Brasileiro de Psiquiatria (9), Revista Avaliação Psicológica (4), Revista Estudos de Psicologia (2) Revista de Saúde Publica (2), Psicologia em Estudo (2). Revista CEFAC (2) e Revista Ciência e Saúde Coletiva (2)

Com relação à natureza dos estudos observou-se que são predominantemente quantitativos totalizando 24 artigos. Os estudos de cunho qualitativos foram dezesseis demostrando assim uma diferença considerável dentre o material analisado neste trabalho. Além desses foram encontrados três artigos de revisão bibliográfica e um de estudo de caso 
evidenciando assim o foco de estudo atual sobre os sintomas depressivos na infância e sua mensuração.

Considerando os objetivos dos trabalhos analisados, foi possível identificar, por meio da sistematização das temáticas presentes, que trabalhos sobre a incidência dos sintomas depressivos em crianças e estudos sobre depressão infantil e suas comorbidades foram os mais encontrados. A Tabela 1 evidencia esses dados apresentando ainda outros temas presentes nos objetivos dos artigos aqui estudados.

Tabela 1. Temáticas dos artigos e termos indicativos correspondentes.

\begin{tabular}{|c|c|c|}
\hline Objetivos & Termos Indicativos & $\mathbf{N}$ \\
\hline $\begin{array}{l}\text { Analisar variáveis ambientais } \\
\text { associadas a depressão }\end{array}$ & $\begin{array}{l}\text { "Analisar a associação entre variáveis do contexto } \\
\text { familiar e o risco de problemas } \\
\text { emocionais/comportamentais em crianças". }\end{array}$ & 15 \\
\hline Validar instrumentos & $\begin{array}{l}\text { "Analisar os parâmetros psicométricos da versão } \\
\text { brasileira reduzida do CDI". }\end{array}$ & 7 \\
\hline Verificar Incidência & $\begin{array}{l}\text { "Verificar a incidência de sintomas depressivos em } \\
\text { uma amostra de crianças". }\end{array}$ & 7 \\
\hline $\begin{array}{l}\text { Recolher representações da } \\
\text { criança sobre a depressão }\end{array}$ & $\begin{array}{l}\text { "Apreender as representações sociais da depressão } \\
\text { elaboradas por crianças inseridas no Ensino } \\
\text { Fundamental". }\end{array}$ & 2 \\
\hline $\begin{array}{l}\text { Verificar relações entre } \\
\text { depressão e rendimento } \\
\text { escolar }\end{array}$ & $\begin{array}{l}\text { "Relações entre emoções e saúde de alunos com e } \\
\text { sem dificuldade de aprendizagem". }\end{array}$ & 6 \\
\hline Identificar comorbidades & $\begin{array}{l}\text { "Identificar prevalência de depressão e de } \\
\text { ansiedade" }\end{array}$ & 7 \\
\hline
\end{tabular}

Partindo das informações acima citadas fica evidenciada uma preocupação com a verificação das associações entre os contextos de inserção das crianças e a incidência da depressão. Nessa direção cabe esclarecer que os contextos mais pesquisados foram o institucional (9) e o escolar (6). Os trabalhos analisados com o intuito de verificar ou estudar as comorbidades relacionadas à depressão na infância também foram significativos representando sete artigos. Sendo assim, a Tabela 2 evidencia quais foram as comorbidades estudadas juntamente com o tema da depressão infantil e a frequência com que elas apareceram nas análises.

Tabela 2. Depressão Infantil e comorbidades

\begin{tabular}{lc}
\hline Comorbidades & Incidência \\
\hline Obesidade Infantil & 4 \\
Ansiedade & 3 \\
Disturbios do sono & 3 \\
Estresse & 3 \\
Transtorno Bipolar & 1 \\
\hline
\end{tabular}

\section{DISCUSSÃO}

Verificou-se que os trabalhos aqui recuperados não foram publicados em um único periódico embora tenha ocorrido predominância em periódicos da área médica. Paralelamente a estas constatações verificou-se que os trabalhos de cunho quantitativo representaram $54,5 \%$ do total de artigos analisados, já os estudos qualitativos somaram $36,3 \%$ dos trabalhos, revelando uma discrepância dentre as publicações sobre a depressão em crianças, onde a maioria dos estudos publicados apresenta a realização de pesquisas estatísticas e quantitativas. Ainda na análise do tipo de estudo realizado pelos artigos recuperados constatou-se que somente um dos 
artigos era de estudo de caso e quatro trabalhos de revisão bibliográfica. Aqui se abre uma maior possibilidade de desenvolvimento de novos trabalhos, de modo particular dentro das pesquisas qualitativas, que favoreça uma compreensão mais ampla da depressão em crianças a fim de possibilitar avanços diagnósticos e terapêuticos adequados ao período de desenvolvimento em que criança se encontra.

Fica, pois, claro que, entre os materiais recuperados, a preocupação com a elaboração de diagnósticos precisos. A esse respeito, discutiram-se quais instrumentos podem ser eficazes. Wathier (2008) em seu estudo trouxe os resultados que corroboram a validade da adaptação do Inventário de Depressão Infantil (CDI) para a população brasileira demostrando sua importância na construção do diagnóstico de depressão infantil como sendo um instrumento importante neste cenário.

Na mesma direção, Coutinho et al. (2008) e Cruvinel et al. (2008a) realizaram estudos de validação e análise psicométrica do CDI visando torná-lo um instrumento que alcance os objetivos propostos em sua versão original auxiliando na identificação da depressão principalmente por meio da informação fornecida pela própria criança.

Estudos sobre a incidência dos sintomas depressivos em crianças em outros ambientes como o familiar e escolar também foram analisados neste trabalho. Nesse sentido Cruvinel e Boruchovitch (2008) identificaram, por meio da aplicação do CDI, em sua versão original e na adaptada para o Brasil, o número de crianças que apresentavam sintomas de depressão em duas amostras de estudantes da cidade de Campinas - SP. O resultado apontou que a prevalência de sintomatologia depressiva no primeiro estudo foi de $3,55 \%$, e, no segundo estudo de $17 \%$.

Como já foi dito, a análise do material resgatado permitiu ainda identificar os trabalhos que tratavam o tema da depressão infantil e suas comorbidades. Apareceram relacionadas a obesidade, ansiedade, stress, doenças físicas e distúrbios do sono como principais comorbidades. Tais observações possibilitam compreensão da importância de se obter uma avaliação precisa da presença de sintomas depressivos na criança no sentido de permitir um diagnóstico diferencial, e ainda entender o modo como os sintomas tiveram inicio e se existe relação com outra patologia que se encontre como gênese ou consequência de tais sintomas.

\section{CONCLUSÃO}

Nos estudos sobre depressão infantil analisados neste trabalho, temáticas como a de validação de instrumentos, incidência de depressão em crianças, variáveis ambientais associadas à depressão, relações entre depressão e rendimento escolar e comorbidades são marcantes e se mostram expressivas. As peculiaridades com que a depressão se manifesta na criança fomentam estudos que visam, tanto por meio do aprofundamento do conceito como pelo desenvolvimento de instrumentos precisos e eficazes, um melhor diagnóstico e prognóstico na intenção de aumentar a capacidade de compreensão da patologia e suas implicações no desenvolvimento da criança.

Em relação aos contextos nos quais a depressão foi estudada, houve predominantemente o interesse pelos contextos institucionais. Isso permite inferir que existe uma preocupação subjacente com a não patologização da criança, ou seja, a depressão enquanto um fenômeno construído pode ser considerada. No entanto, é importante salientar que os dados extraídos da produção científica realizada na ultima década sobre a depressão infantil revelam inúmeras possibilidades de pesquisas nesta área de modo especial em pesquisas qualitativas, estudos de caso e revisão bibliográfica que apareceram de forma menos expressiva nos artigos analisados. 


\section{REFERÊNCIAS}

AMERICAN PSYCHIATRIC ASSOCIATION. DSM-5: manual diagnóstico e estatístico de transtornos mentais. 5. ed. Porto Alegre: Artmed, 2014. 992p.

BAHLS, Saint-Clair. Aspectos clínicos da depressão em crianças e adolescentes: clinical features. J. Pediatr. (Rio J.), Porto Alegre, v. 78, n. 5, p. 359-366, Oct. 2002 . Available from $<$ http://www.scielo.br/scielo.php?script=sci_arttext\&pid=S0021-

$75572002000500004 \&$ Ing=en\&nrm=iso>. access on 29 July 2017. http://dx.doi.org/10.1590/S0021-75572002000500004,

COUTINHO, Maria da Penha de Lima; CAROLINO, Zulmira Carla Gonçalves; MEDEIROS, Emerson Diógenes de. Inventário de Depressão Infantil (CDI): evidências de validade de constructo e consistência interna. Aval. psicol., Porto Alegre, v. 7, n. 3, p. 291-300, dez. 2008 . Disponível em <http://pepsic.bvsalud.org/scielo.php?script=sci_arttext\&pid=S1677-

$04712008000300004 \&$ Ing=pt\&nrm=iso>. acessos em 29 jul. 2017.

CRUVINEL, Miriam; BORUCHOVITCH, Evely; SANTOS, Acácia Aparecida Angeli dos. Inventário de Depressão Infantil (CDI): análise dos parâmetros psicométricos. Fractal, Rev. Psicol., Rio de Janeiro , v. 20, n. 2, p. 473-489, Dec. 2008 . Available from $<$ http://www.scielo.br/scielo.php?script=sci_arttext\&pid=S1984-

02922008000200013\&lng=en\&nrm=iso>. access on 29 July 2017. http://dx.doi.org/10.1590/S1984-02922008000200013,

CRUVINEL, Miriam; BORUCHOVITCH, Evely. Sintomas depressivos em crianças: estudos com duas versões do CDI. Psicol. cienc. prof., Brasília , v. 28, n. 3, p. 574-585, 2008 . Available from $<$ http://www.scielo.br/scielo.php?script=sci_arttext\&pid=S1414-

98932008000300011\&lng=en\&nrm=iso>.

access

on 29 July 2017. http://dx.doi.org/10.1590/S1414-98932008000300011,

CRUVINEL, Miriam; BORUCHOVITCH, Evely. Regulação emocional em crianças com e sem sintomas de depressão. Estud. psicol. (Natal), Natal, v. 16, n. 3, p. 219-226, Dec. 2011 . Available from $<$ http://www.scielo.br/scielo.php?script=sci_arttext\&pid=S1413-

294X2011000300003\&lng=en\&nrm=iso>.

access

on 29 July 2017. http://dx.doi.org/10.1590/S1413-294X2011000300003.

KOVACS, M.. Children Depression Inventory CDI: Manual. New York: Multi- Health Systems. 1992. LIMA, Dênio. Depressão e doença bipolar na infância e adolescência. J. Pediatr. (Rio J.), Porto Alegre , v. 80, n. 2, supl. p. 11-20, Apr. 2004 . Available from <http://www.scielo.br/scielo.php?script=sci_arttext\&pid=S0021-

$75572004000300003 \&$ Ing=en\&nrm=iso>.

access

on 29 July 2017. http://dx.doi.org/10.1590/S0021-75572004000300003,

PAPALIA, D. E. Desenvolvimento Humano. 10a edição, Artmed, São Paulo. 2009.

SANTOS, Patricia Leila dos. Problemas de saúde mental de crianças e adolescentes atendidos em um serviço público de psicologia infantil. Psicol. estud., Maringá , v. 11, n. 2, p. 315321, Aug. 2006 Available from <http://www.scielo.br/scielo.php?script=sci_arttext\&pid=S1413-

$73722006000200010 \&$ Ing=en\&nrm=iso>. access on 29 July 2017. http://dx.doi.org/10.1590/S1413-73722006000200010. 
WATHIER, Josiane Lieberknecht; DELL'AGLIO, Débora Dalbosco; BANDEIRA, Denise Ruschel. Análise fatorial do Inventário de Depressão Infantil (CDI) em amostra de jovens brasileiros. Aval. psicol., Porto Alegre , v. 7, n. 1, p. 75-84, abr. 2008 . Disponível em <http://pepsic.bvsalud.org/scielo.php?script=sci_arttext\&pid=S1677$04712008000100010 \&$ lng=pt\&nrm=iso>. acessos em 29 jul. 2017.

WORLD HEALTH ORGANIZATION. HEALTH TOPICS. Depression. Recuperado em 17 de jun. 2017: http://www.who.int/topics/depression/en/. 